\title{
Valoración clínica del dolor en unidades de cuidados intensivos neonatales españolas
}

\author{
Clinical assessment of pain in spanish neonatal intensive care units
}

\author{
Alejandro Avila-Alvarez ${ }^{\mathrm{a}}$, Ricardo Carbajal $^{\mathrm{b}}$, Emilie Courtois ${ }^{\mathrm{b}}$, Sonia Pertega- \\ Diaz $^{c}$, Kanwaljeet J.S. Anand ${ }^{\mathrm{d}}$, Javier Muñiz-Garcia ${ }^{\mathrm{e}}$, Grupo español del proyecto \\ Europain
}

\author{
${ }^{a}$ Unidad de Neonatología, Servicio de Pediatría, Instituto de Investigación Biomédica de A Coruña \\ (INIBIC), Complexo Hospitalario Universitario de A Coruña (CHUAC), SERGAS, Universidade de A \\ Coruña (UDC), A Coruña, España \\ ${ }^{b}$ Service d'Urgences Pédiatriques. Hôpital d'enfants Armand Trousseau, Inserm UMR 1153 Equipe de \\ recherche en Epidémiologie Obstétricale, Périnatale et Pédiatrique (EPOPé), Université Pierre et Marie \\ Curie, París, Francia \\ ${ }^{c}$ Grupo de Investigación de Epidemiología Clínica y Bioestadística, Instituto de Investigación Biomédica de \\ A Coruña (INIBIC), Complexo Hospitalario Universitario de A Coruña (CHUAC), SERGAS, Universidade \\ de A Coruña (UDC), A Coruña, España \\ ${ }^{d}$ University of Tennessee Health Science Center, Memphis, Estados Unidos \\ ${ }^{e}$ Instituto Universitario de Ciencias de la Salud e Instituto de Investigación Biomédica de A Coruña \\ (INIBIC), Complexo Hospitalario Universitario de A Coruña (CHUAC), Sergas, Universidade de A Coruña \\ (UDC), A Coruña, España
}

\begin{abstract}
Resumen
Introducción. Las escalas clínicas son hoy en día el mejor método para evaluar el dolor en el neonato, dada la imposibilidad de autorreporte en este grupo de edad. Se diseñó un estudio con el objetivo de determinar las prácticas actuales en relación con la valoración clínica del dolor en España y los factores asociados al uso de escalas clínicas.

Métodos. El estudio es de tipo observacional, longitudinal y prospectivo. Participaron 30 unidades y se reclutó a 468 neonatos.

Resultados. Solo 13 unidades (43,3\%) disponían de protocolos de valoración del dolor. Se evaluó el dolor con una escala en 78 neonatos $(16,7 \%$, IC del $95 \%, 13,1-20,1)$ y el número medio de valoraciones del dolor por paciente y día de estancia fue de $2,3 \pm 4,8$, con una mediana de 0,75 . Del total de 7.189 días-paciente estudiados, $654(9,1 \%)$ conllevaron al menos una valoración del dolor. Veinte unidades $(66,7 \%)$ no realizaron evaluación del dolor con una escala clínica en ningún paciente. Entre las que sí lo hicieron, se observó una gran variabilidad en el porcentaje de pacientes en los que se evaluó el dolor y en las escalas utilizadas. La escala CRIES (C-Crying; R-Requires increased oxygen administration; I-Increased vital signs; E-Expression; S-Sleeplessness) fue la que se usó en más unidades. En el análisis multivariante solo la ventilación mecánica invasiva se asoció a recibir valoración del dolor con una escala (OR 1,46, p=0,042).

Discusión. La mayoría de los neonatos ingresados en cuidados intensivos en España no recibe una valoración del dolor. Muchas unidades todavía no utilizan rutinariamente las escalas clínicas y entre las que las utilizan existe una gran variabilidad. Estos resultados pueden servir de base para la elaboración de guías nacionales al respecto del dolor en el neonato.
\end{abstract}




\begin{abstract}
Introduction. Clinical scales are currently the best method to assess pain in the neonate, given the impossibility of self-report in this age group. A study is designed with the aim of determining the current practices as regards the clinical assessment of pain in Spanish Neonatal Units and the factors associated with the use of clinical scales.

Methods. A prospective longitudinal observational study was conducted. A total of 30 Units participated and 468 neonates were included.

Results. Only 13 Units (43.3\%) had pain assessment protocols. Pain was evaluated with a scale in 78 neonates $(16.7 \%, 95 \% \mathrm{CI} ; 13.1-20.1)$ and the mean number of pain assessments per patient and per day was 2.3 (Standard Deviation; 4.8), with a median of 0.75 . Of the total number of 7,189 patient-days studied, there was at least one pain assessment in $654(9.1 \%)$. No pain assessment was performed with a clinical scale on any patient in $20(66.7 \%)$ Units. Among those that did, a wide variation was observed in the percentage of patients in whom pain was assessed, as well as in the scales used. The CRIES (C-Crying; R-Requires increased oxygen administration; I-Increased vital signs; E-Expression; S-Sleeplessness) scale was that used in most Units. In the multivariate analysis, only invasive mechanical ventilation was associated with receiving a pain assessment with a scale (OR 1.46, $\mathrm{P}=.042)$.

Discussion. The majority of neonates admitted into Intensive Care in Spain do not receive a pain assessment. Many units still do not routinely use clinical scales, and there is a wide variation between those that do use them. These results could serve as a basis for preparing national guidelines as regards pain in the neonate.
\end{abstract}

\title{
Palabras clave
}

Neonato, Dolor, Sedación, Analgesia

Keywords

Neonate, Pain, Sedation, Analgesia

\section{Introducción}

El manejo del dolor es una prioridad de los cuidados intensivos neonatales. De una forma simplificada, se puede decir que el adecuado manejo del dolor en neonatología se sustenta en cuatro puntos fundamentales: 1) la reducción del número de procedimientos potencialmente dolorosos; 2) el uso de medidas no farmacológicas; 3) el uso de fármacos analgésicos, y 4) la valoración del dolor con escalas clínicas ${ }^{1,2}$. El autorreporte, que constituye el patrón de oro de la valoración del dolor a partir de la edad escolar, es, por razones obvias, imposible en el neonato y esta incapacidad probablemente ha influido en el infratratamiento histórico del dolor en neonatología ${ }^{3}$. En un intento de objetivar la presencia o ausencia de dolor y de medir su intensidad, se han desarrollado escalas clínicas que combinan una serie de parámetros fisiológicos y conductuales.

Aunque hay varias revisiones recientes al respecto ${ }^{3-6}$, los estudios específicos sobre la frecuencia y el tipo de valoración del dolor en la práctica clínica en neonatología son escasos. La mayoría de los estudios que estiman el uso de escalas clínicas son estudios tipo encuesta en los que se pregunta a las unidades sobre sus pautas generales al respecto del manejo del dolor ${ }^{7-9}$.

Hasta donde conocemos, en España no existen actualmente datos sobre cómo se valora el dolor en las unidades de cuidados intensivos neonatales (UCIN). Dentro del proyecto internacional europeo Europain, diseñamos un estudio específico de la muestra española con el objetivo de determinar las prácticas clínicas actuales en relación con la valoración clínica del dolor sobre la base de la proporción de recién nacidos que reciben valoración del dolor con una escala clínica, las escalas que son utilizadas y el número de veces que se utilizan. Además, intentamos identificar factores asociados al uso de escalas clínicas y al número de valoraciones. 


\section{Material y métodos}

El estudio es de tipo observacional, longitudinal y prospectivo. Una descripción más detallada de los métodos se puede consultar en el estudio del manejo farmacológico del dolor recientemente publicado $^{10}$. Participaron 30 unidades neonatales distribuidas por todo el país (anexo 1). En cada unidad participante se incluyó a todos los recién nacidos que ingresaron durante un mes (noviembre del 2012) hasta una edad corregida de 44 semanas. De cada neonato se recogieron datos demográficos, de los modos de asistencia respiratoria, del uso de sedantes o analgésicos y de la valoración de dolor con escalas clínicas. La duración de la recogida de datos para cada recién nacido fue de 28 días o hasta el alta. Se obtuvo la aprobación de la Agencia Española de Medicamentos y Productos Sanitarios y del comité de ética de investigación clínica de referencia.

Se realizó un análisis descriptivo de las características de las unidades participantes y de los pacientes incluidos. Las variables cuantitativas se expresan como media \pm desviación estándar, mediana, rango intercuartílico y/o rango. Para las variables cualitativas, se calculó la distribución de frecuencias y porcentajes.

Se analizaron las variables asociadas a la valoración del dolor con una escala clínica (si o no) y al número de valoraciones del dolor por paciente y día de estancia en aquellos neonatos en los que sí se usó una escala. En el análisis bivariado, se utilizó el test de la chi al cuadrado o el test exacto de Fisher según procediese. Para variables numéricas, se utilizó el test de Mann-Whitney.

Posteriormente, para determinar las variables asociadas de forma independiente a la valoración del dolor, se realizó un modelo de regresión logística. Se siguió una estrategia de modelización hacia delante, introduciendo en el modelo todas aquellas variables que en el análisis bivariado mostraron un valor de $\mathrm{p}<0,1$. El análisis estadístico se realizó con el programa SPSS 19.0 para Windows. Todos los tests se realizaron con un planteamiento bilateral. Se consideraron significativos valores de $\mathrm{p}<0,05$.

\section{Resultados}

De las 30 unidades neonatales que participaron, 20 (66,6\%) disponían de guías locales de tratamiento del dolor y $13(43,3 \%)$ disponían de guías locales de valoración del dolor. En 6 unidades $(20 \%)$ había designado un líder del dolor médico y en 4 (13.3\%) un líder del dolor en enfermería. En 13 unidades (43,3\%) existía un equipo del dolor. En la tabla 1 se describen las características asistenciales (número de camas e ingresos) de las unidades participantes. El porcentaje de inclusión medio de las unidades fue muy alto $(94,79 \%)$ y el número de pacientes incluidos por cada unidad se relacionó directamente con el número de camas (coeficiente de correlación de 0,77 ) y con el número de ingresos anuales (coeficiente de correlación de 0,87 ).

Tabla 1. Características de las unidades participantes

\begin{tabular}{ll}
\hline \multicolumn{2}{c}{ Unidades, $\mathrm{N}=30$} \\
\hline \\
$\begin{array}{l}\text { Número de camas } \\
\text { Media } \pm \mathrm{DE}\end{array}$ \\
$\begin{array}{l}\text { Mediana (rango) } \\
\text { Número de ingresos/año }\end{array}$ & $13,1 \pm 7, .0$ \\
Media \pm DE & $12(2-33)$ \\
Mediana (rango) & $272,9 \pm 137,1$ \\
Número de ingresos quirúrgicos/año & \\
Media \pm DE & $42,9 \pm 54,1$ \\
Mediana (rango) & $25(0-222)$ \\
& \\
\hline
\end{tabular}

DE: desviación estándar. 
Se incluyó a un total de 468 neonatos. Sus características generales se recogen en la tabla 2. Globalmente, 198 neonatos recibieron algún tipo de medicación sedante o analgésica durante el periodo de estudio $(42,3 \%$, IC del $95 \%, 37,7-46,8)$ y 155 neonatos $(33,1 \%$, IC del 95\%, 28,7-37,5) recibieron sedantes o analgésicos mayores (opiáceos, benzodiacepinas, ketamina o propofol).

Tabla 2.Características de los neonatos incluidos, de la muestra total y según la asistencia respiratoria máxima recibida durante el periodo de estudio

\begin{tabular}{|c|c|c|c|c|}
\hline Variable & $\begin{array}{l}\text { Total } \\
\mathrm{N}=468\end{array}$ & $\begin{array}{l}\text { Ventilación mecánica } \\
\text { invasiva } \\
n=202(43,2 \%)\end{array}$ & $\begin{array}{l}\text { Ventilación no invasiva o } \\
\text { respiración espontánea } \\
n=266(56,8 \%)\end{array}$ & $\begin{array}{l}\text { P valor } \\
(\chi 2 \text { o t de } \\
\text { Student })\end{array}$ \\
\hline \multicolumn{5}{|l|}{ Edad gestacional (semanas) } \\
\hline Media $\pm \mathrm{DE}$ & $34,3 \pm 4,6$ & $33,4 \pm 5,2$ & $34,9 \pm 4,0$ & $<0,001$ \\
\hline Mediana (IQ) & $\begin{array}{l}34,3(30,2- \\
38,5)\end{array}$ & $33,9(29,1-38)$ & $34,5(31,6-38,8)$ & - \\
\hline $24-29, \mathrm{n}(\%)$ & $107(22,8)$ & $70(34,7)$ & $37(13,9)$ & $<0,001$ \\
\hline $30-32, \mathrm{n}(\%)$ & $76(16,2)$ & $24(11,9)$ & $52(19,5)$ & \\
\hline $33-36, \mathrm{n}(\%)$ & $115(24,5)$ & $38(18,8)$ & $77(28,9)$ & \\
\hline $37-42, \mathrm{n}(\%)$ & $170(36,3)$ & $70(34,7)$ & $100(37,5)$ & \\
\hline \multicolumn{5}{|l|}{ Peso al nacer (g) } \\
\hline Media $\pm \mathrm{DE}$ & $2.182 \pm 976$ & $2.051 \pm 1.007$ & $2.283 \pm 94$ & 0,011 \\
\hline Mediana (IQ) & $\begin{array}{l}2.081(1.390- \\
3.025)\end{array}$ & $2.050(1.089-2.905)$ & $2.095(1.470-3.090)$ & - \\
\hline Hombre, n (\%) & $256(54,7)$ & $113(55,9)$ & $143(53,7)$ & 0,670 \\
\hline \multicolumn{5}{|l|}{ CRIB score } \\
\hline Media $\pm D E$ & $2,1 \pm 3,0$ & $4 \pm 3,7$ & $0,8 \pm 1,2$ & $<0,001$ \\
\hline Mediana (IQ) & $1(0,0-3,0)$ & $3(1-6)$ & $0(0-1)$ & - \\
\hline $\begin{array}{l}\text { Fallecimiento durante el periodo } \\
\text { de estudio, } \mathrm{n}(\%)\end{array}$ & $21(3,2)$ & $21(10,4)$ & $0(0,0)$ & $<0,001$ \\
\hline
\end{tabular}

CRIB es una medida de la gravedad en neonatos compuesto por 6 ítems recogidos en las primeras $12 \mathrm{~h}$ de vida. Su valor va de 0 a 23 , en donde valores mayores indican mayor gravedad.

DE: desviación estándar; IQ: rango intercuartílico.

Se evaluó el dolor con una escala clínica en 78 de los 468 neonatos (16,7\%, IC del 95\%, 13,120,1). En estos 78 neonatos, el número medio de valoraciones del dolor por paciente durante el periodo de estudio fue de 42,2 $\pm 105,4$, con una mediana (rango intercuartílico) de $10,5(5-30,5)$ y el número medio de valoraciones del dolor por paciente y día de estancia fue de $2,3 \pm 4,8$, con una mediana de $0,75(0,4-1,7)$. Solo $45(22,2 \%)$ de los 202 neonatos que recibieron ventilación mecánica invasiva (VM) y $40(25,8 \%)$ de los 155 neonatos que recibieron sedantes o analgésicos mayores recibieron alguna valoración del dolor.

En la figura 1 se representa el porcentaje de valoración de dolor y de administración de fármacos sedantes o analgésicos en las 30 unidades participantes, observándose una marcada variabilidad de los valores. Veinte $(66,7 \%)$ unidades no realizaron evaluación del dolor con una escala clínica en ningún paciente. Las 10 unidades que realizaron valoración clínica del dolor en al menos uno de los pacientes lo hicieron en un porcentaje variable que osciló entre el 4,3 y el 100\% de los neonatos incluidos en el estudio. 


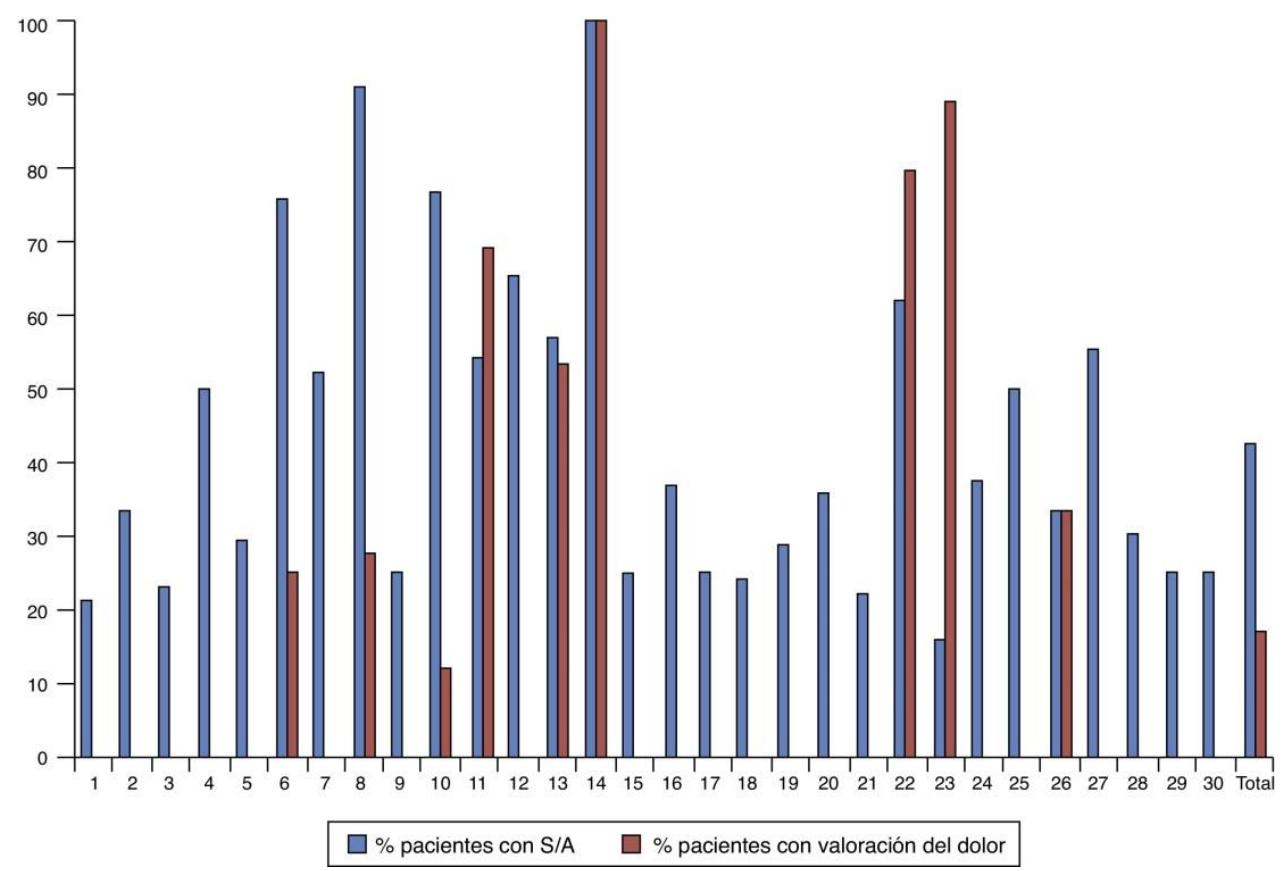

Figura 1.Representación gráfica del uso de los fármacos sedantes y/o analgésicos (S/A) y del uso de escalas clínicas del dolor en cada una de las 30 unidades participantes y en la muestra total sobre la base del porcentaje de pacientes que recibieron fármacos $\mathrm{S} / \mathrm{A}$ o al menos una valoración del dolor.

En estas 10 unidades se usaron 6 escalas clínicas distintas. Las escalas más utilizas fueron la escala NIPS, la escala CRIES y la Susan-Givens. La figura 2 representa el porcentaje de utilización de cada escala sobre el total de neonatos que recibieron alguna valoración del dolor. Por unidades, la escala CRIES se usó en 4 unidades, las escalas PIPP, Susan-Givens y COMFORT en 2 unidades, y las escalas NIPS y N-PASS en una unidad.

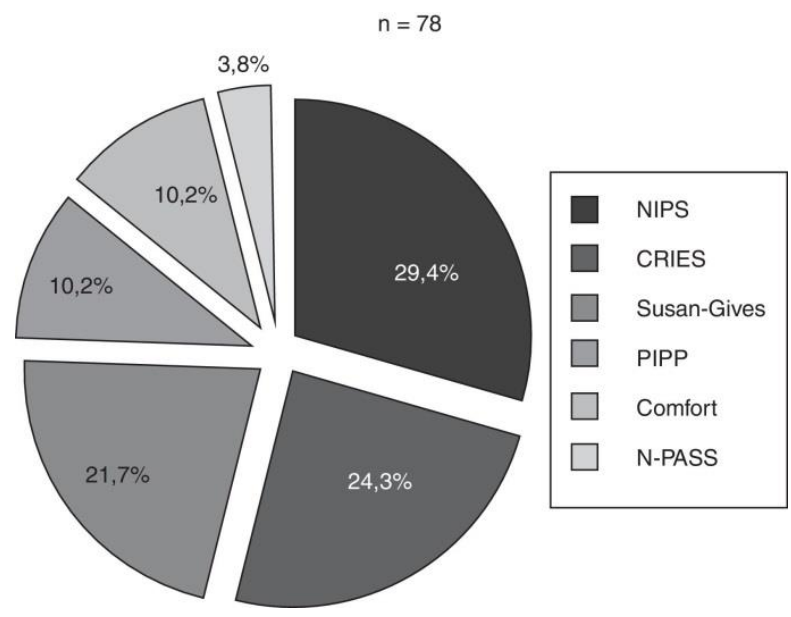

Figura 2.Proporción de neonatos (\%) en los que se usó cada escala sobre el total de neonatos en los que se evaluó el dolor.

CRIES: Crying, Requires Oxygen, Saturation, Increased Vital Signs, Expression and Sleeplessness; NIPS: Neonatal Infant Pain Score; NPASS: Neonatal Pain, Agitation and Sedation Scale; PIPP: Premature Infant Pain Profile. 
El periodo de estudio de los 468 neonatos incluidos representó 7.189 días-paciente, de los cuales $654(9,1 \%)$ conllevaron al menos una valoración del dolor. Se realizó una valoración del dolor en 235/1.000 (23,5\%) días-paciente durante los cuales los neonatos recibieron sedantes o analgésicos mayores y en 419/6.189 (6,8\%) días-paciente sin sedantes o analgésicos mayores, p<0,001. Se realizó una valoración del dolor en 262/1.292 (20,3\%) días-paciente durante los cuales los neonatos recibieron VM y en 392/5.897 (6,6\%) días-paciente sin VM, p<0,001.

Las tablas 3 y 4 muestran las asociaciones entre las variables relativas a los neonatos o a las unidades y la utilización de escalas clínicas de valoración del dolor (si o no) y el número de valoraciones por paciente y día de estancia en aquellos neonatos en los que sí se utilizaron.

Tabla 3.Valoración del dolor con escalas clínicas según las características de la unidad. Análisis bivariante

\begin{tabular}{|c|c|c|c|c|c|c|}
\hline & \multicolumn{2}{|c|}{$\begin{array}{c}\% \text { de neonatos en los que se realizó } \\
\text { evaluación del dolor }\end{array}$} & \multirow[b]{2}{*}{$\mathrm{p}$} & \multicolumn{2}{|c|}{$\begin{array}{c}\mathrm{N} .^{\circ} \text { valoraciones dolor por paciente } \\
\text { y día de estancia }\end{array}$} & \multirow[b]{2}{*}{$\mathrm{p}$} \\
\hline & Media $\pm \mathrm{DE}$ & Mediana & & Media (DT) & Mediana & \\
\hline $\begin{array}{l}\text { Guías locales de } \\
\text { tratamiento del dolor }\end{array}$ & & & 0,350 & & & 0,943 \\
\hline Sí(n=20) & $20,3 \pm 32,8$ & 0 & & $2,5(5,2)$ & 0,7 & \\
\hline No $(n=10)$ & $8,1 \pm 21,8$ & 0 & & $1,2(1,0)$ & 0,7 & \\
\hline $\begin{array}{l}\text { Guías locales de } \\
\text { evaluación del dolor }\end{array}$ & & & $\mathbf{0 , 0 3 5}$ & & & 0,001 \\
\hline Sí (n=13) & $25,7 \pm 33,1$ & 11,8 & & $2,6(5,8)$ & 0,7 & \\
\hline No $(n=17)$ & $9,0 \pm 25,6$ & 0 & & $1,9(1,4)$ & 1,4 & \\
\hline $\begin{array}{l}\text { Líder de dolor médico en } \\
\text { la unidad }\end{array}$ & & & 0,462 & & & $<0,001$ \\
\hline Sí(n=6) & $22,9 \pm 39,9$ & 2,2 & & $14,8(9,5)$ & 19,1 & \\
\hline No $(n=24)$ & $14,6 \pm 27,5$ & 0 & & $1,1(1,1)$ & 0,7 & \\
\hline $\begin{array}{l}\text { Líder de dolor enfermería } \\
\text { en la unidad }\end{array}$ & & & 0,425 & & & $<0,001$ \\
\hline Sí (n=4) & $33,3 \pm 47,1$ & 16,7 & & $17,1(7,8)$ & 20,2 & \\
\hline No $(n=26)$ & $13,6 \pm 26,6$ & 0 & & $1,1(1,1)$ & 0,7 & \\
\hline $\begin{array}{l}\text { Equipo del dolor en la } \\
\text { unidad }\end{array}$ & & & 0,746 & & & 0,860 \\
\hline Sí (n=13) & $19,2 \pm 32,3$ & 0 & & $1,2(1,2)$ & 0,7 & \\
\hline No $(n=16)$ & $8,6 \pm 18,5$ & 0 & & $1,0(0,9)$ & 0,7 & \\
\hline $\begin{array}{l}\text { Visitas de los padres } \\
\text { permitidas } 24 \mathrm{~h}\end{array}$ & & & 0,659 & & & 0,240 \\
\hline Sí (n=26) & $17,8 \pm 31,5$ & 0 & & $2,4(4,9)$ & 0,7 & \\
\hline \multirow[t]{2}{*}{ No $(n=4)$} & $6,2 \pm 12,5$ & 0 & & $0,4(0,1)$ & 0,4 & \\
\hline & & Correlación & $\mathrm{p}$ & \multicolumn{2}{|c|}{ Spearman's rho } & $\mathrm{p}$ \\
\hline $\begin{array}{l}\text { Número de camas en la } \\
\text { unidad }\end{array}$ & & 0,359 & 0,051 & $-0,338$ & 0,002 & \\
\hline $\begin{array}{l}\text { Número de ingresos } \\
\text { anuales }\end{array}$ & & 0,265 & 0,156 & $-0,137$ & 0,230 & \\
\hline $\begin{array}{l}\text { Número de ingresos } \\
\text { quirúrgicos anuales }\end{array}$ & & 0,007 & 0,970 & $-0,444$ & $<0,001$ & \\
\hline
\end{tabular}

DE: desviación estándar; DT: desviación típica.

Se presentan en negrita los valores de $\mathrm{p}<0.05$ 
Tabla 4.Variables del paciente asociadas a la valoración del dolor con escalas clínicas. Análisis bivariante

\begin{tabular}{|c|c|c|c|c|c|c|}
\hline & \multicolumn{3}{|c|}{ Valoración del dolor } & \multicolumn{2}{|c|}{$\mathrm{N}^{\circ}$ valoraciones dolor/día estancia } & \multirow[b]{3}{*}{$\mathrm{p}$} \\
\hline & Sí & No & & & & \\
\hline & $\mathrm{N}(\%)$ & $\mathrm{N}$ & $\mathrm{p}$ & Media $\pm \mathrm{DE}$ & Mediana & \\
\hline Total & $78(16,7)$ & $390(83,3)$ & & $2,3 \pm 4,8$ & 0,75 & \\
\hline Edad gestacional & & & 0,405 & & & 0,009 \\
\hline 24-29 semanas & $17(15,8)$ & $90(84,2)$ & & $5,6 \pm 8,2$ & 1,4 & \\
\hline 30-32 semanas & $8(10,5)$ & $68(89,5)$ & & $2,8 \pm 6,6$ & 0,4 & \\
\hline 33-36 semanas & $22(19,1)$ & $93(80,9)$ & & $0,8 \pm 0,7$ & 0,7 & \\
\hline $37-42$ semanas & $31(18,2)$ & $139(81,8)$ & & $1,5 \pm 2,3$ & 0,8 & \\
\hline Prematuridad $(\leq 36$ semanas) & & & 0,492 & & & 0,507 \\
\hline Sí & $47(15,7)$ & $251(84,3)$ & & $2,9 \pm 5,9$ & 0,7 & \\
\hline No & $31(18,2)$ & $139(81,8)$ & & $1,5 \pm 2,3$ & 0,8 & \\
\hline Ventilación mecánica invasiva & & & 0,005 & & & 0,063 \\
\hline Sí & $45(22,2)$ & $157(77,8)$ & & $3,2 \pm 6,2$ & 0,8 & \\
\hline No & $33(12,4)$ & $233(87,6)$ & & $1,1 \pm 1,3$ & 0,6 & \\
\hline Medicación sedante/analgésica & & & 0,001 & & & 0,310 \\
\hline Sí & $46(23,2)$ & $152(76,8)$ & & $3,1 \pm 6,1$ & 0,7 & \\
\hline No & $32(11,8)$ & $238(88,2)$ & & $1,2 \pm 1,3$ & 0,7 & \\
\hline Sedantes/analgésicos mayores ${ }^{a}$ & & & $<0,001$ & & & 0,453 \\
\hline Sí & $40(25,8)$ & $115(74,2)$ & & $2,5 \pm 5,2$ & 0,7 & \\
\hline No & $38(12,1)$ & $275(87,9)$ & & $2,1 \pm 4,5$ & 0,7 & \\
\hline CRIB score & & & 0,199 & & & 0,181 \\
\hline$\leq 3$ & $58(15,5)$ & $315(84,5)$ & & $2,1 \pm 4,6$ & 0,7 & \\
\hline$>3$ & $20(21,1)$ & $75(78,9)$ & & $2,9 \pm 4,8$ & 0,7 & \\
\hline
\end{tabular}

CRIB es una medida de la gravedad en neonatos compuesto por 6 ítems recogidos en las primeras $12 \mathrm{~h}$ de vida. Su valor va de 0 a 23, en donde valores mayores indican mayor gravedad.

DE: desviación estándar.

${ }^{a}$ Opiáceos, benzodiacepinas, ketamina o propofol.

Además, para la variable valoración del dolor con una escala clínica (sí o no), se ajustó un modelo de regresión logística. Ajustando por la medicación sedante o analgésica, por la existencia de guías locales de evaluación del dolor y por el número de camas en la unidad, solo la VM se asoció de forma significativa a recibir al menos una valoración del dolor con una escala clínica ( $\mathrm{p}=0,042$, OR 1,46, IC del 95\%, 1,01-2,11).

\section{Discusión}

Hasta donde nosotros conocemos, este es el primer estudio multicéntrico prospectivo con respecto a la valoración del dolor en las UCIN españolas. Estos datos complementan aquellos referentes al manejo farmacológico de la sedación y analgesia recientemente publicados en esta revista $^{10,11}$. La unión de ambos trabajos ofrece una visión global del manejo del dolor en las UCIN españolas. El presente estudio demuestra una baja utilización de las escalas clínicas de valoración del dolor, así como una importante variabilidad entre las unidades. Tan solo el 16,7\% de los 468 neonatos ingresados en cuidados intensivos recibió al menos una valoración del dolor con una escala clínica. Solamente un 23,5 y un 20,3\% de días-paciente con administración de sedantes o analgésicos mayores y con VM, respectivamente, conllevaron una valoración del dolor. Es destacable también que 20 de las 30 unidades participantes no realizaron evaluación del dolor con 
una escala clínica en ningún paciente, y que en las unidades en las que sí se evaluó el dolor existen diferencias importantes en el número de evaluaciones realizadas y en las escalas clínicas utilizadas.

Es difícil comparar nuestros resultados con otros estudios, ya que son muy escasos los trabajos que, como el presente, recogen de forma prospectiva datos con pacientes reales «a pie de cuna» sobre el uso de escalas de valoración del dolor. Roofthooft et al., en un estudio holandés centrado en el dolor asociado a procedimientos, describen su experiencia tras la introducción de una serie de medidas de mejora del control del dolor, consiguiendo que prácticamente la totalidad de los pacientes $(96,6 \%)$ reciban una valoración del dolor durante el periodo de estudio ${ }^{12}$.

La literatura muestra sobre todo estudios tipo encuesta a las unidades. Por ejemplo, en una encuesta realizada a 16 UCIN francesas en el año 2002, el 60\% declaró usar escalas para el dolor agudo y el $53 \%$ para el dolor prolongado ${ }^{7}$. En este estudio, las principales razones esgrimidas para no usar escalas fueron (por este orden): el desconocimiento, la falta de consideración del dolor como una prioridad, la creencia de que las escalas no son válidas y la falta de tiempo. Probablemente, estas razones son aplicables al día a día de cualquier unidad. En una encuesta a 90 unidades neonatales italianas, solo el $19 \%$ usaba escalas para valorar el dolor o la analgesia durante la VM ${ }^{9}$. En Australia, en el año 2005, solo el 6\% de 105 unidades declaró usar escalas de valoración del dolor ${ }^{8}$, y 6 años después el porcentaje ascendió tan solo a un $11 \%{ }^{13}$. En este estudio, de forma similar al nuestro, también se encontraron diferencias importantes entre regiones del mismo país. En España, la encuesta desarrollada en unidades de intensivos pediátricas en el año 2011 ya reflejó que más de la mitad de las unidades no usaban rutinariamente escalas para monitorizar la sedoanalgesia ${ }^{14}$.

Es destacable que en las 10 unidades que sí valoraron el dolor con escalas clínicas se usaron 6 escalas distintas (fig. 2). En una encuesta a 370 unidades neonatales de Austria, Alemania y Suiza solo 32 declararon usar escalas de valoración del dolor y estas usaron hasta 19 escalas distintas ${ }^{15}$. La valoración del dolor con escalas es una recomendación ampliamente difundida por distintas sociedades científicas y documentos de consenso ${ }^{1}$, pero el hecho de que existan múltiples escalas publicadas y validadas no es sinónimo de que hayamos encontrado la manera ideal de evaluar el dolor $^{3}$. La valoración debe corresponder al tipo de dolor y existen un dolor agudo de corta duración creado por un procedimiento y un dolor prolongado que corresponde a un estado de persistencia del estímulo nocivo o de la lesión tisular. Sin embargo, la mayoría de las escalas están desarrolladas para el dolor agudo ${ }^{5,16}$. Entre las escalas usadas en nuestro estudio, la NIPS y la PIPP son para el dolor agudo de procedimientos, la CRIES para el dolor postoperatorio y las escalas COMFORT y N-PASS para el dolor prolongado de la VM. No conocemos referencias publicadas de validación de la escala Susan-Givens.

$\mathrm{Al}$ analizar las razones que pueden haber contribuido a una baja utilización de las escalas en las unidades españolas, probablemente haya influido que actualmente no se pueda recomendar una sola escala clínica como la ideal sobre las otras ${ }^{4,6}$. Además, la incorporación de las escalas a la práctica clínica es difícil y requiere entrenamiento ${ }^{17}$. También suponen un aumento de la carga de trabajo y muchas veces su registro no se acompaña de una acción médica determinada, ya que existen pocos protocolos de tratamiento asociados a escalas clínicas ${ }^{18}$ o algunos de los propuestos son de difícil aplicación práctica ${ }^{3}$. Deindl et al. han comunicado su experiencia en 2 unidades austríacas en las que la implementación de un protocolo de manejo del dolor basado en la escala N-PASS conllevó un aumento del uso de opiáceos sin afectar al tiempo de VM ni de estancia hospitalaria ${ }^{18}$. Desde hace una década se ha difundido el concepto de que el dolor es el quinto signo vital y que como tal debe figurar en las historias clínicas. Sin embargo, la valoración que conduce al diagnóstico de la presencia del dolor, pero que solo figura en la historia clínica del paciente sin que sirva para adecuar el tratamiento no es útil y probablemente tampoco ética. Existen algunas recomendaciones para adecuar la respuesta terapéutica analgésica a la valoración del dolor ${ }^{19} \mathrm{y}$, por ejemplo, se ha recomendado que el uso de opiáceos en neonatos sometidos a VM se haga de forma selectiva sobre la base de medidas clínicas del dolor ${ }^{20}$. Somos conscientes de que la ausencia de uso de escalas de valoración del dolor en algunas unidades no implica que en esas unidades no se esté tratando el dolor o no se esté evaluando de forma no reglada. Las escalas 
pretenden homogeneizar y sistematizar la valoración del dolor y con ello aportar cierto grado de objetividad.

Con respecto a los factores asociados al uso de escalas clínicas de valoración del dolor, el análisis bivariado (tablas 3 y 4) mostró para el uso de las escalas (sí o no) una asociación significativa con el hecho de recibir VM, con el uso de medicación sedante/analgésica, con el uso de analgésicos/sedantes mayores y con la existencia de guías locales de evaluación del dolor, aunque en el análisis multivariante solo la VM mantuvo significación. En el análisis bivariado, se asociaron a un menor número de valoraciones el número de camas en la unidad y el número de ingresos quirúrgicos, lo que podría hablarnos de la influencia negativa de la carga de trabajo sobre el uso de las escalas. Solo la existencia de guías locales de evaluación del dolor se asoció tanto al hecho de usar las escalas de valoración, como al número de valoraciones por día de estancia. Este hallazgo es concordante con lo observado previamente por otros estudios ${ }^{9,15}$ y de nuevo reafirma la importancia de los protocolos de manejo del dolor. De hecho, se ha recomendado ampliamente que cada unidad establezca sus propias guías de valoración del dolor y que esta valoración esté asociada a una respuesta terapéutica determinada ${ }^{2,3,9}$. Parece lógico concluir que la presencia de guías a nivel nacional podría conllevar beneficios similares y que la homogeneización de las pautas facilitaría la comparación de resultados y la evaluación del impacto de los cambios sobre las pautas de sedación y analgesia.

Este estudio tiene ciertas limitaciones. Primero, la generalización de los resultados podría verse afectada por el hecho que algunas unidades españolas no han participado. Sin embargo, la gran mayoría de las unidades invitadas participaron, con representación de unidades de todo el país e inclusión de un número elevado de pacientes consecutivos no seleccionados, por lo que creemos que constituyen un reflejo fiel de la situación real. Segundo, no se puede descartar que el hecho de participar en el estudio, por saberse observado, modifique las prácticas. Se intentó minimizar este aspecto con un tiempo relativamente largo de inclusión (un mes) y de seguimiento (28 días), que garantiza la rotación del personal. Tercero, el diseño del estudio no permite obtener información sobre los valores de las escalas del dolor ni sobre las consecuencias clínicas para el neonato de esta evaluación. Consideramos que la inclusión de estos datos causaría una carga muy importante sobre el personal o los investigadores, y podría haber reducido de manera importante la participación de los centros o la exhaustividad.

\section{Conclusiones}

Este estudio demuestra que la mayoría de los neonatos ingresados en las UCIN españolas no recibe una valoración del dolor con una escala clínica y que muchas unidades todavía no utilizan las escalas clínicas de forma rutinaria. Además, existe una gran variabilidad entre las UCIN españolas en relación con la valoración del dolor. Creemos que estos resultados indican la necesidad de establecer guías de práctica clínica a nivel nacional al respecto del manejo del dolor neonatal.

\section{Financiación}

Este estudio ha recibido financiación del Séptimo Programa Marco de la Unión Europea (beca 223767) dentro del proyecto NeoOpioid.

\section{Conflicto de intereses}

Los autores declaran no tener ningún conflicto de intereses. 


\begin{abstract}
Anexo 1
Grupo español estudio EUROPAIN

Helena Viana, Paloma Lopez Ortego (Hospital Infantil Universitario La Paz, Madrid). Pilar Saenz Gonzalez, Raquel Escrig (Hospital Universitari i Politecnic La Fe, Valencia). Eva Bargalló Ailagas, Concepcio Carles (Hospital Universitari Josep Trueta, Girona). Laura San Feliciano, Ana Belén Mateo (Hospital Universitario de Salamanca). Inés Esteban Diéz, Rosa González Crespo (Hospital San Pedro de Logroño). Ersilia González Carrasco, Isabel de la Nogal Tagarro (Hospital Universitario Severo Ochoa, Leganés). María Dolores Elorza Fernández, Nerea Benito Guerra (Hospital Universitario de Donostia). Salud Luna Lagares, Pedro Jiménez Parrilla (Hospital Virgen Macarena, Sevilla). Francesc Botet, Anna Ciurana, Rebeca Tarjuelo (Hospital Clínic de Barcelona). María José García Borau, Nuria Ibáñez (Hospital de la Santa Creu i Sant Pau, Barcelona). Gloria Diáñez Vega (Hospital Universitario Puerta del Mar, Cádiz). María Arriaga Redondo (Hospital General Universitario Gregorio Marañón, Madrid). Gloria Herranz, Virginia de la Fuente Iglesias (Hospital Clínico San Carlos, Madrid). Amaya Pérez Ocón, Sagrario Santiago Aguinaga (Complejo Hospitalario de Navarra). Belén Martín Parra, Antonia Valero Cardona (Hospital General de Castellón). María Dolores Sánchez-Redondo, Antonio Arroyos Plana (Hospital Virgen de la Salud, Toledo). Zenaida Galve Pradel, Nuria Clavero Montañés (Hospital Universitario Miguel Servet, Zaragoza). María Jesús Ripalda Crespo, Raquel Nogales Juárez (Hospital Universitario Príncipe de Asturias, Alcalá de Henares). Aintzane Euba Lopez, Sonia Fernández de Retana (Hospital Universitario de Álava). Mar Reyné Vergeli, Raquel Vidal (Hospital Sant Joan de Déu, Barcelona). Jose Luis Fernandez-Trisac, María Taboada (Complexo Hospitalario Universitario de A Coruña). Aurora Montoro Expósito, Fátima Camba Longueira (Hospital Vall d $\mathrm{d}_{\zeta}$ Hebron, Barcelona). Gonzalo Solís Sánchez (Hospital Universitario Central de Asturias, Oviedo). María Purificación Ventura Faci, Marivi Mallen (Hospital Clínico Universitario Lozano Blesa, Zaragoza). Pilar Crespo Suárez, Elvira de Sola (Complexo Hospitalario de Pontevedra). Caridad Tapia Collados (Hospital General de Alicante). Isabel de las Cuevas, Beatriz Martín (Hospital Universitario Marqués de Valdecilla, Santander). María Luz Couce Pico, Alejandro Pérez Muñuzuri, Salomé Quintáns (Complexo Hospitalario Universitario de Santiago). Ana Melgar Bonis, Eugenia Bodas (Hospital Universitario 12 de Octubre, Madrid). Ana Concheiro Guisan, Begoña Pérez Costas (Complexo Hospitalario Universitario de Vigo).
\end{abstract}

\title{
Bibliografía
}

1. D.G. Batton,K.J. Barrington,C. Wallman. Prevention and management of pain in the neonate: An update. Pediatrics., 118 (2006), pp. 2231-2241.

2. K.J. Anand. Consensus statement for the prevention and management of pain in the newborn. Arch Pediatr Adolesc Med., 155 (2001), pp. 173-180.

3. P. Hummel,M. van Dijk. Pain assessment: Current status and challenges. Semin Fetal Neonatal Med., 11 (2006), pp. 237-245.

4. M. Ranger,C.C. Johnston,K.J.S. Anand. Current controversies regarding pain assessment in neonates. Semin Perinatol, 31 (2007), pp. 283-288.

5. M. Van Dijk,D. Tibboel. Update on pain assessment in sick neonates and infants pain measurement critically ill neonates infants review, 59 (2012), pp. 1167-1181.

6. K.J.S. Anand. Pain assessment in preterm neonates. Pediatrics, 119 (2007), pp. 605-607.

7. T. Debillon,V. Bureau,C. Savagner,V. Zupan-Simunek,R. Carbajal. Pain management in French neonatal intensive care units. Acta Paediatr, 91 (2002), pp. 822-826.

8. D. Harrison,P. Loughnan,L. Johnston. Pain assessment and procedural pain management practices in neonatal units in Australia. J Paediatr Child Health, 42 (2006), pp. 6-9.

9. P. Lago,A. Guadagni,D. Merazzi,G. Ancora,C.V. Bellieni,A. Cavazza. Pain management in the neonatal intensive care unit: A national survey in Italy. Paediatr Anaesth, 15 (2005), pp. 925-931.

10. A. Avila-Alvarez,R. Carbajal,E. Courtois,S. Pertega-Diaz,J. Muñiz-Garcia,K.J.S. Anand. [Sedation and analgesia practices among Spanish neonatal intensive care units]. An Pediatr (Barc), 83 (2015), pp. 75-84.

11. M. Eriksson. [Good news for Spanish neonates]. An Pediatr (Barc), 83 (2015), pp. 73-74.

12. D.W.E. Roofthooft,S.H.P. Simons,K.J.S. Anand,D. Tibboel,M. van Dijk. Eight years later, are we still hurting newborn infants?. Neonatology, 105 (2014), pp. 218-226.

13. J. Foster,K. Spence,D. Henderson-Smart,D. Harrison,P.H. Gray,J. Bidewell. Procedural pain in neonates in Australian hospitals: A survey update of practices. J Paediatr Child Health, 49 (2013), pp. E35-E39.

14. S. Mencía,M. Botrán,J. López-Herce,J. del Castillo. [Sedative, analgesic and muscle relaxant management in Spanish paediatric intensive care units]. An Pediatr (Barc), 74 (2011), pp. 396-404.

15. B. Gharavi,C. Schott,M. Nelle,G. Reiter,O. Linderkamp. Pain management and the effect of guidelines in neonatal units in Austria, Germany and Switzerland. Pediatr Int, 49 (2007), pp. 652-658.

16. L.J. Duhn,J.M. Medves. A systematic integrative review of infant pain assessment tools. Adv Neonatal Care, 4 (2004), pp. 126-140.

17. K.J.S. Anand,J.V. Aranda,C.B. Berde,S. Buckman,E.V. Capparelli,W. Carlo. Summary proceedings from the neonatal pain-control group. Pediatrics, 117 (2006), pp. S9-S22. 
18. P. Deindl,L. Unterasinger,G. Kappler,T. Werther,C. Czaba,V. Giordano. Successful implementation of a neonatal pain and sedation protocol at 2 NICUs. Pediatrics., 132 (2013), pp. e211-e218.

19. S.H.P. Simons,M. van Dijk,K.S. Anand,D. Roofthooft,R.A. van Lingen,D. Tibboel. Do we still hurt newborn babies? A prospective study of procedural pain and analgesia in neonates. Arch Pediatr Adolesc Med, 157 (2003), pp. 1058-1064.

20. R. Bellù,K. de Waal,R. Zanini. Opioids for neonates receiving mechanical ventilation: A systematic review and meta-analysis. Arch Dis Child Fetal Neonatal Ed, 95 (2010), pp. F241-F251. 